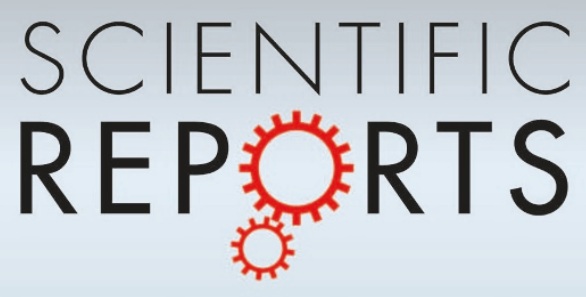

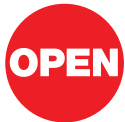

SUBJECT AREAS:

BIOMATERIALS

CARBON NANOTUBES AND

FULLERENES

TOXICOLOGY

ONCOGENESIS

Received

1 May 2012

Accepted

22 June 2012

Published

9 July 2012

Correspondence and requests for materials should be addressed to N.S. (saitoko@shinshuu.ac.jp)

* These authors contributed equally to this work.

\section{Carcinogenicity evaluation for the application of carbon nanotubes as biomaterials in rasH2 mice}

\author{
Seiji Takanashi ${ }^{*}$, Kazuo Hara ${ }^{*}$, Kaoru Aoki ', Yuki Usui ${ }^{2}$, Masayuki Shimizu', Hisao Haniu', \\ Nobuhide Ogihara', Norio Ishigaki' ', Koichi Nakamura' ', Masanori Okamoto', Shinsuke Kobayashi', \\ Hiroyuki Kato' ${ }^{1}$ Kenji Sano ${ }^{3}$, Naoyuki Nishimura ${ }^{4}$, Hideki Tsutsumi ${ }^{5}$, Kazuhiko Machida ${ }^{5}$ \& Naoto Saito ${ }^{6}$
}

'Department of Orthopaedic Surgery, Shinshu University School of Medicine, Asahi 3-1-1, Matsumoto, Nagano 390-8621, Japan, ${ }^{2}$ Department of Research Center for Exotic Nanocarbons, Shinshu University School of Engineering, Wakasato 4-17-1, Nagano, Nagano 380-8553, Japan, ${ }^{3}$ Department of Laboratory Medicine, Shinshu University Hospital, Asahi 3-1-1, Matsumoto, Nagano 390-8621, Japan, ${ }^{4}$ R\&D Center, Nakashima Medical Co. Ltd., Haga 5322, Kita-ku, Okayama 701-1221, Japan, ${ }^{5}$ Testing Department, Central Institute for Experimental Animals, Tonomachi 3-25-12, Kawasaki-ku, Kawasaki, Kanagawa $210-0821$, Japan, ${ }^{6}$ Department of Applied Physical Therapy, Shinshu University School of Health Sciences, Asahi 3-1-1, Matsumoto, Nagano 390-8621, Japan

The application of carbon nanotubes (CNTs) as biomaterials is of wide interest, and studies examining their application in medicine have had considerable significance. Biological safety is the most important factor when considering the clinical application of CNTs as biomaterials, and various toxicity evaluations are required. Among these evaluations, carcinogenicity should be examined with the highest priority; however, no report using transgenic mice to evaluate the carcinogenicity of CNTs has been published to date. Here, we performed a carcinogenicity test by implanting multi-walled CNTs (MWCNTs) into the subcutaneous tissue of ras $\mathrm{H} 2$ mice, using the carbon black present in black tattoo ink as a reference material for safety. The rasH2 mice did not develop neoplasms after being injected with MWCNTs; instead, MWCNTs showed lower carcinogenicity than carbon black. Such evaluations should facilitate the clinical application and development of CNTs for use in important medical fields.

arbon nanotubes (CNTs) are fibrous particles with nanoscale diameter and microscale length. CNTs have unique physical and chemical characteristics, and the study and development of these particles is ongoing in various fields ${ }^{1-6}$. For example, CNTs have already been used in the electrodes of lithium batteries and displays because of their superior electrical properties ${ }^{7-9}$, as well as in tennis rackets and golf clubs, to take advantage of their good mechanical properties. Furthermore, applications in airplanes and motor vehicle parts are expected in the future ${ }^{10,11}$. In the medical field, the use of CNTs as biomaterials has been extensively studied in recent years ${ }^{12-14}$. CNTs have been reported to be an excellent scaffold material for bone and nerve regeneration ${ }^{15-17}$, and studies examining the efficacy of combining CNTs with existing implants as a reinforcement to improve their mechanical strength and durability are ongoing ${ }^{18}$. CNTs also have applications in cancer therapy, including in drug delivery systems and cancer cell imaging, and the ability of CNTs to absorb electromagnetic waves make them suitable for use in hyperthermia in cancer ${ }^{19,20}$.

Because of their expected applications in various fields, such as those described above, the biological safety of CNTs has been studied worldwide. However, most previous studies have examined the inhalation of CNTs, as this provides the most convenient method of internalization, and studies on the safety of the inhaled particles are urgently required ${ }^{21-23}$. Because CNTs have a long, thin, fibrous structure similar to that of asbestos, which can cause malignant mesothelioma when inhaled into the lungs, CNTs have been suspected to have carcinogenic properties $^{24}$. Various studies examining this possibility have been performed ${ }^{25-28}$; because the results have shown that CNTs and asbestos have different safety characteristics, a different standard value for the safe inhalation of CNTs has been proposed ${ }^{29,30}$.

As opposed to inhalation, the application of CNTs as a biomaterial requires completely different evaluations of safety. Such evaluations include implantation tests, cytotoxicity tests, carcinogenicity tests, and in vivo pharmacokinetic studies $^{31}$. Although some evaluations of the safety of CNTs when used as a biomaterial have been 
published in the last several years, the number of these reports is quite small compared to that for inhalation ${ }^{32}$. The carcinogenicity of CNTs is the most important issue to be addressed when considering them as a biomaterial, and tests using experimental animals are important for studying carcinogenicity in humans. However, to the best of our knowledge, no evaluations examining the implantation of CNTs in transgenic animals with increased susceptibility to carcinogenic substances have been performed to date.

The most representative transgenic mouse models with increased sensitivity to carcinogens are the $\mathrm{p}^{5} 3^{+/-}$and rasH2 mouse models ${ }^{33}$. While rasH2 mice have been used in previous carcinogenicity studies on biomaterials, these tests examined solid materials, and no study involving particles has been performed to date. The positive and negative controls used for the evaluation of solid materials are $N$-methyl- $N$-nitrosourea (MNU) and high-density polyethylene ${ }^{34}$. However, while MNU can be used as a positive control when evaluating the carcinogenicity of particles, a reference standard of particles that have been established to have no carcinogenicity should be used as the reference negative control ${ }^{26}$.

Asbestos is often used as a control material in carcinogenicity tests of CNTs. However, asbestos is only an appropriate control in inhalation tests, and is not appropriate as a control for the carcinogenicity of implantable materials because it has never been used as a biomaterial. As toxicity due to inhalation and implantation are completely different, it is not possible to evaluate the carcinogenicity of CNTs using asbestos as a reference. We therefore applied carbon black, which is used as a black tattoo ink and has been histologically proven to have no carcinogenicity to the human body, as the most appropriate reference for evaluating the carcinogenicity of CNTs used as biomaterials ${ }^{35}$ in this subcutaneous administration study using rasH2 mice. Our results have important implications on further studies examining the applicability of CNTs as a biomaterial.

\section{Results}

CNTs, reference material and positive control. The CNTs used for evaluation were multi-walled CNTs (MWCNTs) (VGCF-S; Showa Denko, Tokyo, Japan) with a mean diameter of $100 \mathrm{~nm}$, mean length of $10 \mu \mathrm{m}$, and a $>99.9 \%$ carbon content; they were manufactured by chemical vapor deposition. Sterilization was performed by autoclaving at $121^{\circ} \mathrm{C}$ for $15 \mathrm{~min}$. Tween 80 (Polysorbate 80; NOF, Tokyo, Japan) was used as a surfactant to disperse the MWCNTs uniformly in solution.

The shape of precipitated solid contents obtained by drying commercially available black tattoo ink (Sumi-Black; Unique Tattoos, Subiaco, Australia) was nearly uniform, with a diameter of 30-50 nm. The tattoo ink particles contained $99.57 \mathrm{wt} \% \mathrm{C}$ and 0.43 wt $\% \mathrm{Na}$, with no other element being detected. Comparative Raman and transmission electron microscope studies showed that the black tattoo inks were composed of nano-sized carbon black particles. Therefore, the carbon black particles from the black tattoo inks were used as the reference material ${ }^{35}$. The CNT mixture was created by sonicating a physiological saline solution containing 0.1 vol\% Tween 80 and $3 \mathrm{mg} / \mathrm{mL}$ MWCNTs for $30 \mathrm{~min}$ by ultrasonic dispersion; the reference mixture was created from carbon black ( $3 \mathrm{mg} / \mathrm{mL}$ ) using the same procedure. A positive control solution with known tissue toxicity was prepared by mixing MNU (Wako, Osaka, Japan) with Tween 80 to obtain a $3 \mathrm{mg} / \mathrm{mL}$ solution.

Carcinogenicity study using transgenic rasH2 mice. CNT safety was examined by analyses involving subcutaneous implantation in rasH2 mice (CLEA Japan, Tokyo, Japan), which express the c-Haras proto-oncogene derived from humans. Test substances were implanted when the mice were 6 weeks old, and tumor onset was evaluated after 26 weeks (at 32 weeks of age) to predict the carcinogenicity of these substances in humans. This test period was chosen because rasH2 mice seldom develop spontaneous tumors until they are 34 weeks old ${ }^{27}$.

Six-week-old male rasH2 mice were anesthetized using diethyl ether, and $550 \mu \mathrm{L}$ of CNT mixture (approximately $75 \mathrm{mg} / \mathrm{kg}$ ) was injected into the subcutaneous tissue of the back of each mouse using a 1-mL syringe and a $26 \mathrm{G}$ needle (CNT group). The carbon black, solvent, and MNU groups were injected with the corresponding substances by using the same procedure. Each group consisted of $10 \mathrm{rasH} 2$ mice (total 40 ), and all the mice were weighed once every week during the survival period, except at 10 and 20 weeks, when no and incorrect measurement was performed, respectively. Mice were euthanized after 26 weeks and dissected. Subjects that died or were euthanized because of extreme weakness before 26 weeks were dissected at the time of death.

All the mice in the CNT group and solvent group were alive at 26 weeks after receiving the injection. In the carbon black group, 1 mouse died at 22 weeks, while the 9 remaining mice survived until 26 weeks. In the MNU group, 1 mouse each died at 12, 14, 17, and 21 weeks following injection, while the remaining 6 mice were alive at 26 weeks (Fig. 1a). Statistically significant differences in survival were observed between the MNU and CNT groups, and between the MNU and control groups ( $\mathrm{p}=0.029406$ for both comparisons). When weight change during the study period was examined, the CNT group, carbon black group, and solvent group showed similar changes, while the 4 mice that died prior to the end of the study in the MNU group showed rapid decrease in weight from several weeks before the death. (Fig. 1b).

Histological examination showed a mass in the spleen of 1 mouse from the CNT group; however, it was histologically shown to be an inflammatory pseudotumor (Fig. 2a). The single mouse that died prior to 26 weeks in the carbon black group did not show any neoplasms, and the cause of death was unknown. Of the 9 surviving mice, 1 had a hemangioma in the spleen, while 1 other mouse was found to have a neoplasm in the lung, which was histologically judged to be an adenoma (Fig. 2b). None of the mice in the solvent group were found to have onset of neoplasm in any organ. In contrast, all 10 mice in the MNU group developed neoplasms, including forestomach tumors, papillomas of the skin (face, thigh, and injection site), and perineal tumors in 10, 6, and 5 mice, respectively. In addition, a lung adenoma was observed in 1 mouse, and 2 mice were found to have malignant lymphoma (Fig. 2c). Abnormal growth of squamous cells was histologically shown in all the mice in the MNU group.

Spleen tumors from 1 mouse from each of the CB and CNT groups were also examined by immunostaining for factor VIII, and were diagnosed as a hemangioma and inflammatory pseudotumor, respectively (Fig. 3). The type and frequency of neoplasms in each group are shown in Table 1. A significantly larger number of papillomas were observed in the forestomach and perineal in the MNU group compared to the other groups (significant differences at $\mathrm{p}=0.00000541250$ and $\mathrm{p}=.016254$, respectively).

No neoplasms were detected at the injection sites in the CNT group and macrophage-phagocytosed CNTs were observed; furthermore, inflammatory cells such as neutrophils and lymphocytes were not detected in the surrounding tissues (Fig. 4a). Similarly, neoplasms were not observed at the injection sites in the carbon black group, and images of macrophages taken after they had phagocytosed carbon black particles were similar to those for the CNT group (Fig. 4b). The injection site did not show any change in the solvent group, although a skin tumor was observed at the injection site of 2 mice from the MNU group, which was determined to be a squamous cell tumor upon analysis of a tissue image (Fig. 4c).

\section{Discussion}

Although some studies examining the safety of CNTs as a biomaterial have been published, the evaluation of these results is difficult, and 
a

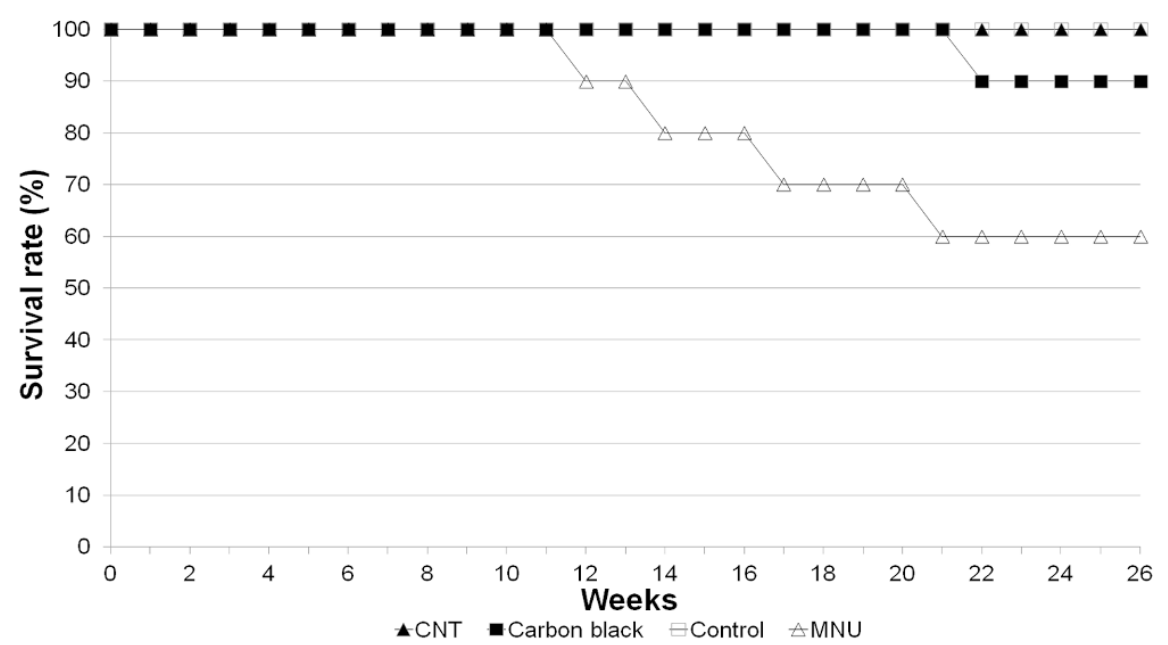

b
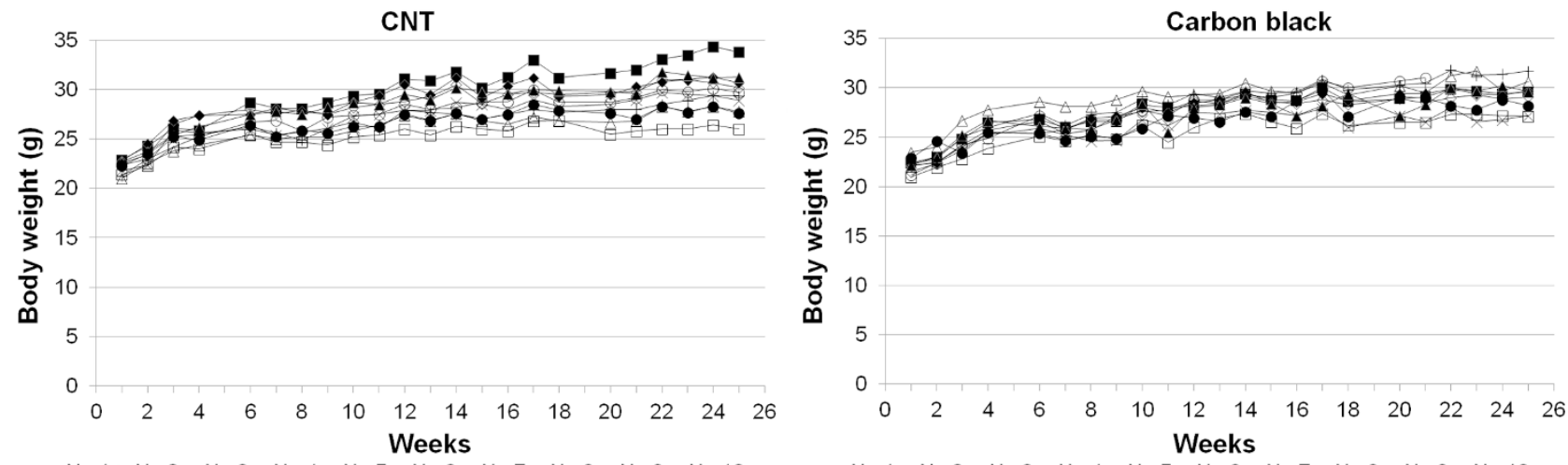

$=\mathrm{No} .1 \boxminus \mathrm{No} 0.2 \bullet \mathrm{No} .3 \bullet \mathrm{No} 0.4 \star \mathrm{No} 0.5 \triangle \mathrm{No} 0.6 \bullet \mathrm{No} .7 \bullet \mathrm{No} 0.8 * \mathrm{No} 0.9+\mathrm{No} .10$

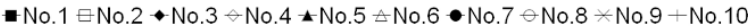
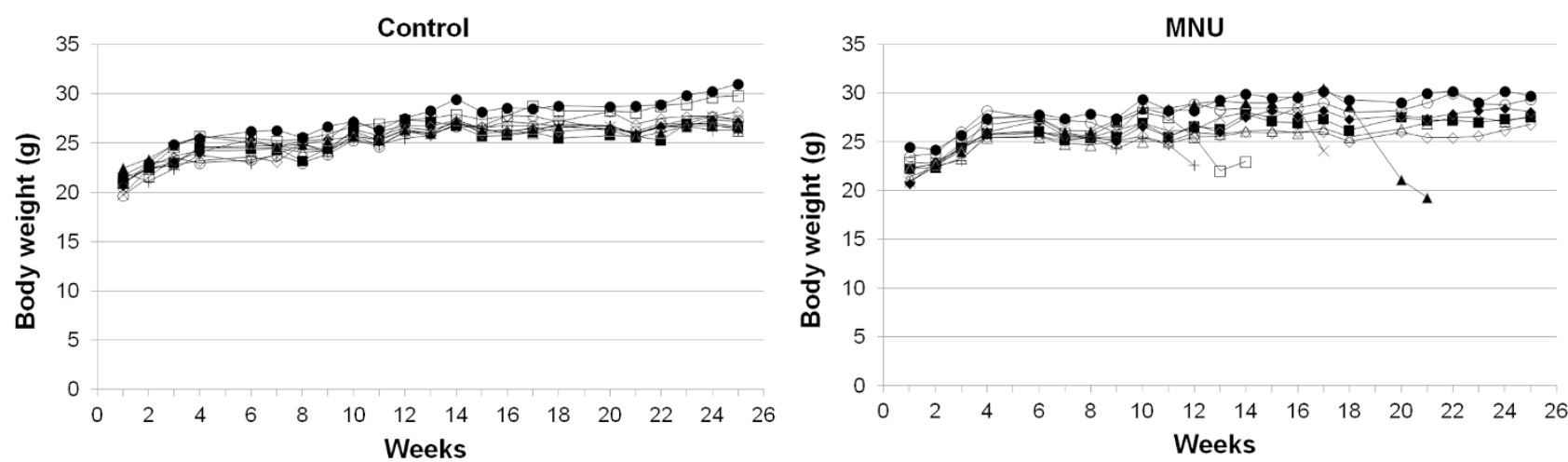

Figure 1 Survival rate and change in the weights of the transgenic rasH2 mice. (a) Survival rate: All the mice in the CNT group and solvent group were alive at 26 weeks after injection. In the carbon black group, 1 mouse died at 22 weeks, and the remaining 9 mice were alive at 26 weeks. In the MNU group, 1 mouse died at each of $12,14,17$, and 21 weeks following the injection, and the remaining 6 mice were alive at 26 weeks. The survival rate was statistically different between the MNU and CNT groups, and between the MNU and control groups ( $p=0.029406$ for both comparisons). (b) Weight change in mice: The CNT group, carbon black group, and solvent group showed similar weight change, while the 4 mice in MNU group that died before the end of the study period showed rapid decrease in weight from several weeks before death.

different results have been reported for the same type of safety test $\mathrm{t}^{37-40}$. The biggest reason for such discrepancy is the lack of a nanoparticle that has established biological safety and is suitable for use as a reference material $^{26}$. Similar to CNTs, which are nanosized carbon particles, tattoo ink is made up of nanosized carbon black particles, and is long known to be safe for use in the human body. In 2011, we therefore reported that black tattoo ink (carbon black) could be an optimal reference material for evaluating the safety of $\mathrm{CNTs}^{35}$. While some studies that used carbon black particles as a negative control for CNTs had been published before our report, the researchers in those studies chose carbon black particles arbitrarily ${ }^{41-45}$. As there are numerous types and grades of carbon black available, a standard for 

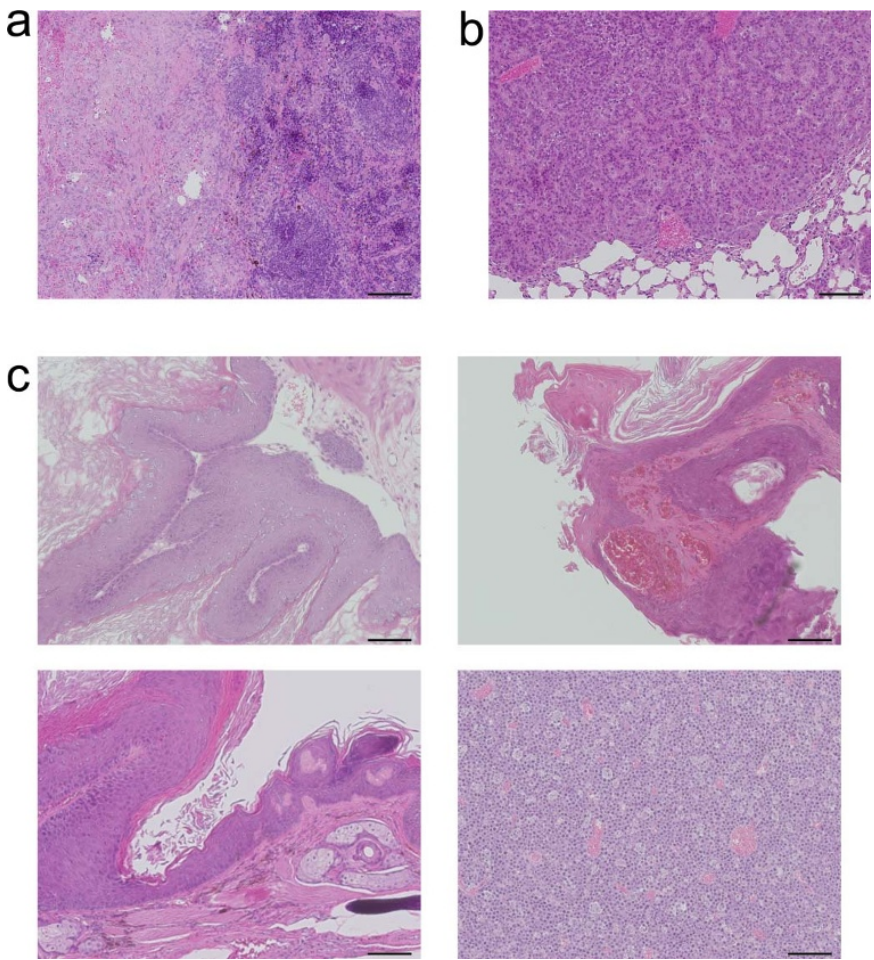

Figure $2 \mid$ Tissue images of masses that developed in the rasH2 mice.

(a) A mass was observed in the spleen of 1 mouse from the CNT group, but it was found to be an inflammatory pseudotumor and not a neoplasm.

(b) One mouse from the carbon black group was alive at the 26th week and was found to have developed a neoplasm in the lung, which was diagnosed as an adenoma. (c) All 10 mice in the MNU group developed forestomach tumors, with abnormal growth of squamous cells (left upper panel).

Papillomas of skin were observed in 6 mice (right upper panel). Perineal tumors were found to have developed in 5 mice (left lower panel), while 2 mice developed malignant lymphoma (right lower panel). Hematoxylineosin staining. Scale bar: $100 \mu \mathrm{m}$.

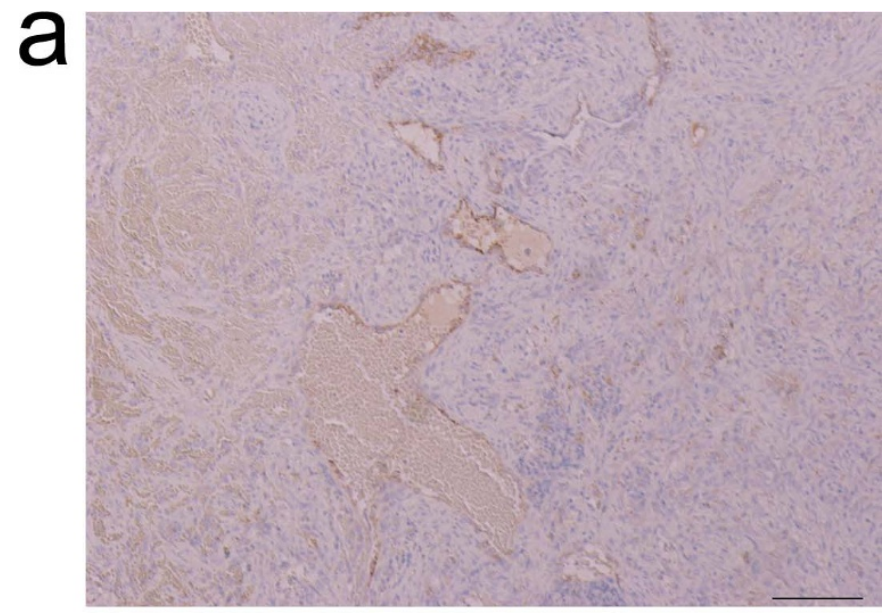

b

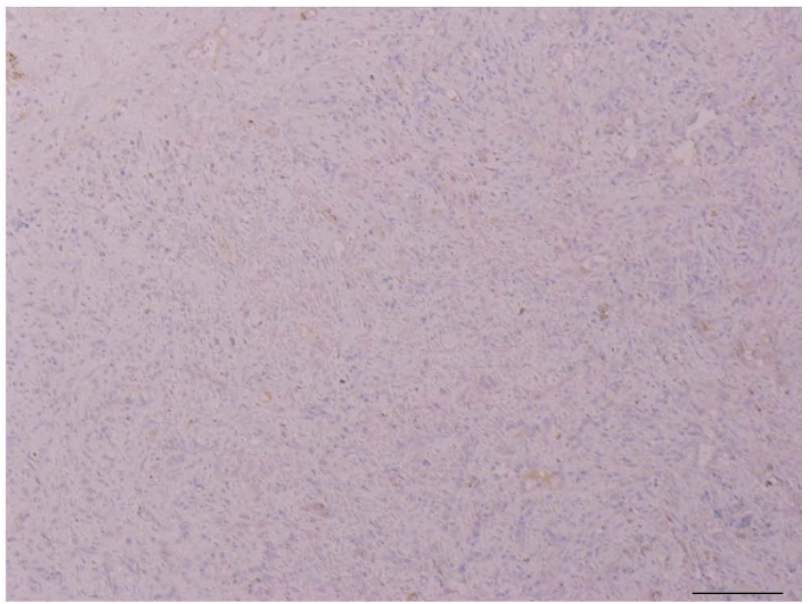

Figure $3 \mid$ Immunostaining of the spleen tissue for Factor VIII.

(a) Hemangioma was diagnosed in the spleen of a mouse from the carbon black group. Immunostaining revealed vascular endothelial-like cells stained in dark brown. Atypical nucleus was not observed.

(b) Inflammatory pseudotumor was diagnosed in the spleen of a mouse from the CNT group. Few cells were stained. Scale bar: $100 \mathrm{~mm}$.

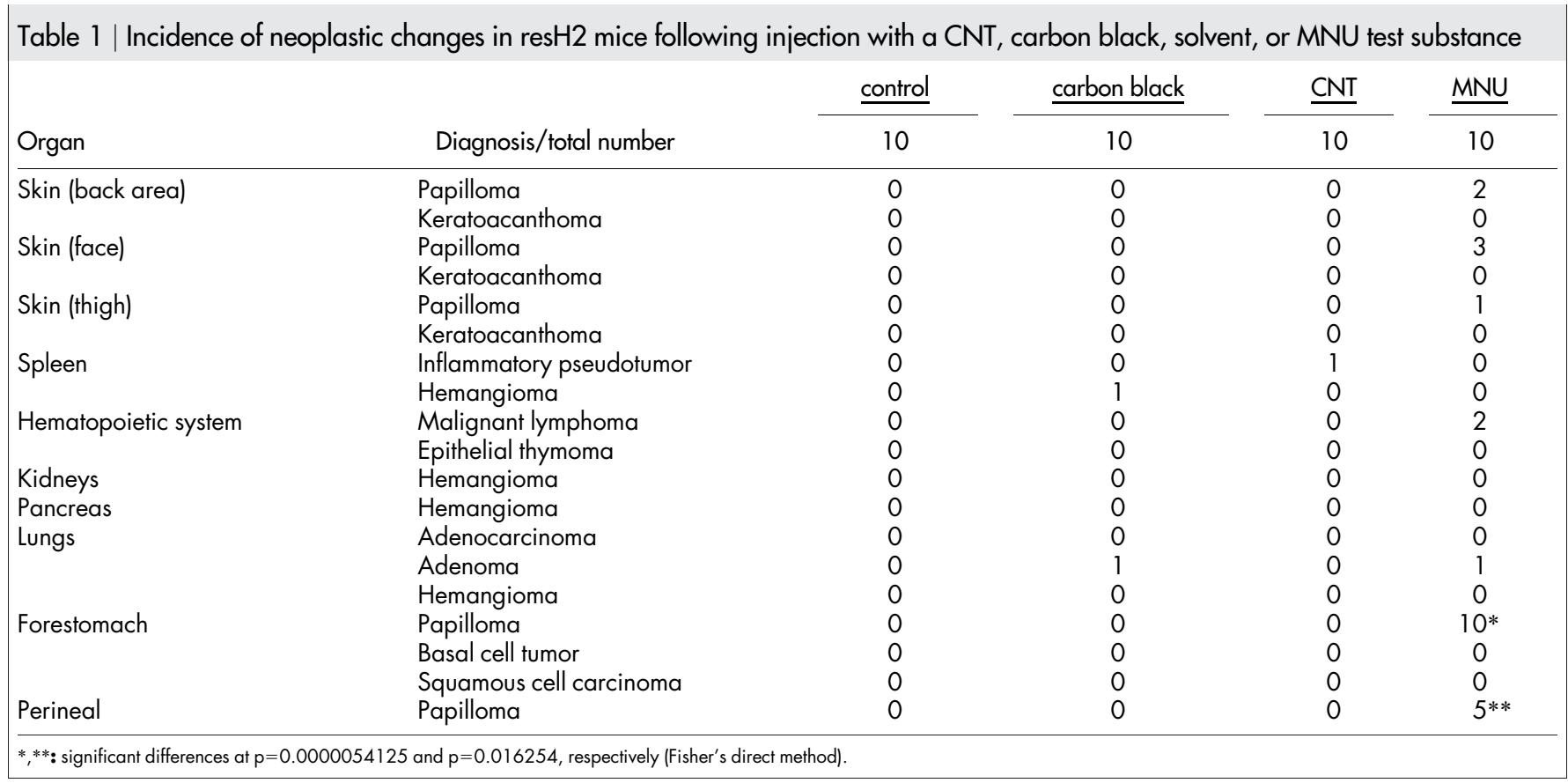


a

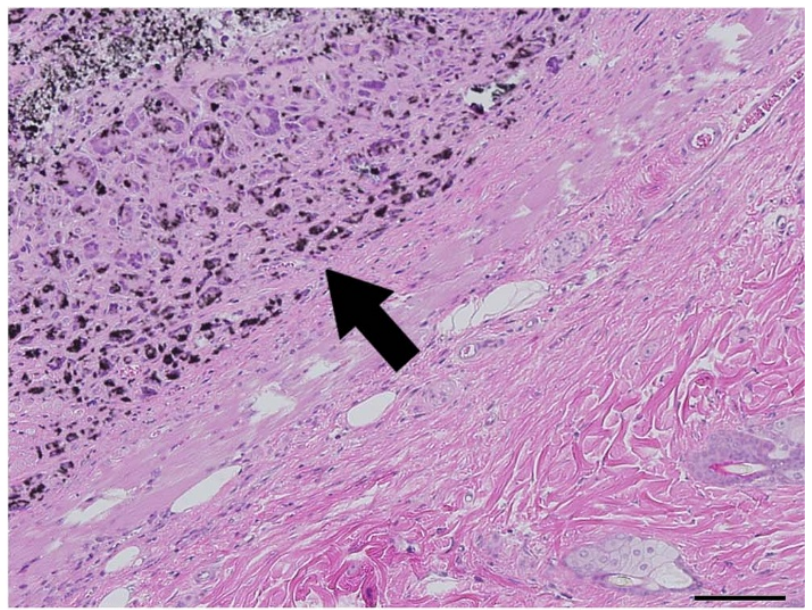

b

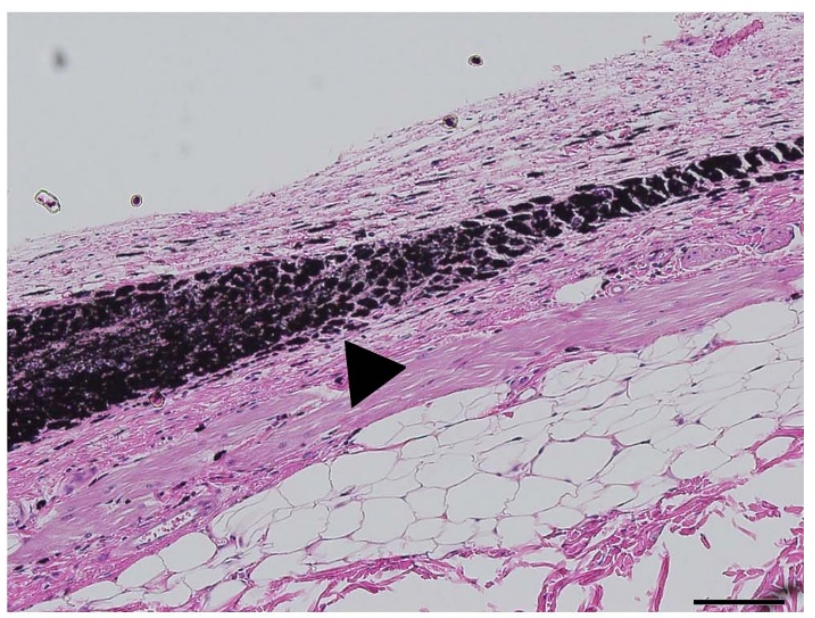

C

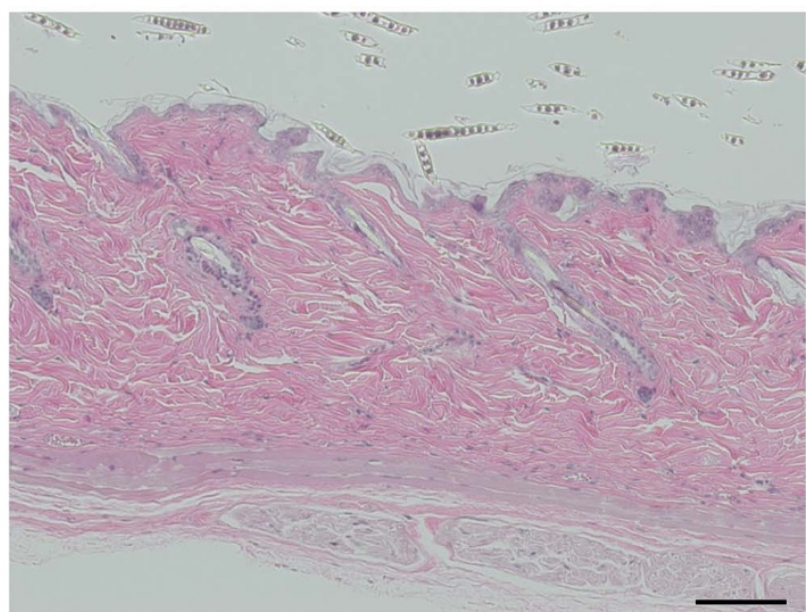

Figure $4 \mid$ Images of the skin and subcutaneous tissue at the injection site of rasH2 mice, at 26 weeks after injection with a CNT, carbon black, or MNU test substance. (a) None of the mice in the CNT group developed neoplasms, and macrophages accumulated after phagocytosing CNT

particles. Inflammatory cells such as neutrophils and lymphocytes were not observed in the surrounding tissues. (b) In the carbon black group, macrophages were similarly found to have phagocytosed carbon black particles, and no neoplasms were observed. (c) In the MNU group, a skin tumor was detected at the injection site of 2 mice, which was found to be a squamous cell tumor. Arrow: CNTs, Arrowhead: carbon black.

Hematoxylin-eosin staining. Scale bar: $100 \mu \mathrm{m}$. its use as a control should be established. We therefore proposed that the optimal standard carbon black should have a carbon purity of $\geq 99.5 \%$ and a particle size of $30-50 \mathrm{~nm}$, similar to that used in tattoo ink $^{35}$. This carbon black and CNTs are both made of highly pure carbon, which allows comparisons at similar weights and enables the quantitative evaluation of CNT safety for use as a biomaterial. While our previous study found that the toxicity of CNTs is comparable to that of the carbon black used in tattoos in an in vivo implantation study, cytotoxicity study, and cytokine production assay of $\mathrm{CNTs}^{35}$, this is the first study to use carbon black as a reference material to examine the subcutaneous implantation of CNTs in test animals susceptible to cancer.

The rasH2 mice used in this study express the human-derived cH-ras proto-oncogene, which causes them to spontaneously develop multiorgan tumors after approximately 35 weeks of age, leading to death. However, these mice seldom develop tumors before 34 weeks of age, and carcinogenicity tests can be performed until then. As this particular characteristic can reduce the test time, work load, and cost of such studies, and the sensitivity of evaluation is high, rasH2 mice are mainly used to evaluate the carcinogenicity of chemical substances that are used in humans ${ }^{46}$. This rasH2 mouse model is approved as a test animal for new drug applications by regulatory authorities such as the Food and Drug Administration in the USA, the Committee for Proprietary Medicinal Products in the EU, and the Ministry of Health, Labour and Welfare in Japan. The company that developed these mice is maintaining this strain in order to preserve study accuracy, so that highly reproducible evaluations can be achieved $^{47,48}$. Although their application in carcinogenesis tests for solid implants of biomaterials has only begun in recent years, such as in tests for the potential carcinogenicity of IC $\operatorname{tags}^{36,48-50}$, the implantation test of CNTs in rasH2 mice reported here is the first to evaluate nanosized particles.

All 10 positive control mice injected with MNU developed neoplasms in 26 weeks, with 4 mice dying prior to the end of the study. In contrast, none of the mice in the CNTs group died; instead, tissue examination of the CNT group mice after dissection showed an inflammatory pseudotumor in only 1 mouse, and neoplasm was not observed in any mouse. The survival rate was significantly different between the MNU and CNT groups. Because the amount of test substance used in this study, i.e., $75 \mathrm{mg} / \mathrm{kg}$, was remarkably higher than that used in conventional mouse implantation tests ${ }^{37-40}$, and the mice used have higher sensitivity to carcinogenicity, we judged that it was not necessary to perform the test with a higher density when neoplasm was not observed. In the carbon black group, a hemangioma in the spleen and neoplasm in the lung of 1 mouse each were also observed. The results from this initial subcutaneous implantation test of carcinogenicity involving transgenic mice with higher susceptibility to cancer have therefore shown that CNTs do not induce the formation of neoplasms, as the carcinogenicity was lower than that of the carbon black used in tattoo ink.

During the majority of carcinogenesis, there is a complex relationship between factors such as the accumulation of mutations, DNA disorders, and chemical changes in nucleotides. Because the occurrence of tumors in transgenic mice such as rasH 2 mice is influenced by both mutated oncogenes and mutated antioncogenes, studies using rasH2 mice can evaluate only the result of the complex process of carcinogenesis ${ }^{35}$. In contrast, genotoxicity tests can evaluate the carcinogenicity of a material for individual parameters, thereby allowing evaluation to be performed relatively easily and the ISO10993 series requires that multiple types of tests be performed ${ }^{31}$. Typical genotoxicity tests include bacterial reverse mutation assays (Ames test), micronucleus assays, and Comet assays. CNTs have already been tested using such methods to examine carcinogenicity following inhalation ${ }^{51-53}$. These genotoxicity tests are also important for the evaluation of carcinogenicity with regard to the application of CNTs as biomaterials, and while many previous reports have found 
that CNTs have no genotoxicity, some have reported differing results, and no consensus has been achieved ${ }^{38,54}$. This discrepancy may result from differences in test conditions, sample size, and evaluation method, although the most important cause is the absence of a suitable reference material ${ }^{26}$. As shown in this study, carbon blacksimilar to that used for tattoo ink-is an appropriate reference material for future studies. The carcinogenicity of CNTs when used as a biomaterial can be further clarified by comprehensive evaluations of the results of genotoxicity tests and implantation tests such as the one performed in this study.

This study is the first to evaluate the carcinogenicity of CNTs as a biomaterial using transgenic rasH2 mice that overexpress the ras $\mathrm{H} 2$ oncogene and are highly sensitive to carcinogenesis. Although CNTs showed no carcinogenicity in this study and had lower carcinogenicity than the reference material, it cannot be completely ruled out on the basis of these results alone. A larger number of carcinogenicity studies comparing the results for CNTs to those for reference materials with established biological safety, such as the carbon black used in this study, should be performed. Results from various genotoxicity studies and studies using test animals susceptive to cancer should also be comprehensively evaluated to judge the carcinogenicity of CNTs. The completion of studies that use CNTs as a biomaterial is expected to result in progress in various important medical fields, including cancer treatment, regenerative medicine, and the development of drug delivery systems.

\section{Methods}

Reference material. The shape of precipitated solid contents obtained by drying commercially available black tattoo ink (Sumi-Black; Unique Tattoos, Subiaco, Australia) was determined by scanning electron microscopy (JSM-7000F and JED-2300, JEOL, Tokyo, Japan) to be nearly uniform, with a diameter of 30-50 nm (Fig. S1a). Elemental and compositional analyses performed using energy dispersive $\mathrm{x}$-ray spectroscopy showed that the tattoo ink particles contained $99.57 \mathrm{wt} \% \mathrm{C}$ and $0.43 \mathrm{wt} \% \mathrm{Na}$, with no other element being detected. Comparative Raman studies (RXN1-532, Kaiser, Ann Arbor, MI) on the tattoo ink particles were performed using the industrial general carbon black (Vulcan XC 72, Cabot, Boston, MA), which is used as a filler for rubber products, as a control. The tattoo ink particles showed a Raman feature similar to that of the general carbon black, where the $\mathrm{G}$ band at $1582 \mathrm{~cm}^{-1}$ originated from the $\mathrm{E}_{2 \mathrm{~g} 2}$ graphite mode and the $\mathrm{D}$ band at $1360 \mathrm{~cm}^{-1}$ was a defectinduced mode (Fig. S1b). The R values (defined as the integrated intensity of the D band divided by the intensity of the $\mathrm{G}$ band) for the tattoo ink particles and general carbon black were comparable. Observation by transmission electron microscope (JEM-2010, JEOL) revealed that the morphologies of the tattoo ink particles were almost the same as that for general carbon black (Fig. S1c).

Carcinogenicity study. Visual inspection of tumor onset in transgenic rasH2 mice was performed during dissection. Each organ was fixed separately, embedded, and cut into thin sections before being stained with hematoxylin and eosin; subsequently, they were placed on a slide and observed using an optical microscope.

As the diagnosis of hemangioma by hematoxylin and eosin staining was difficult in the spleen tissue, vascular endothelial cells were also immunostained for Factor VIII. Two toxicologic pathology specialists diagnosed the tumor in accordance with a globally recognized text of pathological tumor diagnosis in mice (International classification of rodent tumors; the mouse. Editor: Ulrich Mohr. WHO International Agency for Research on Cancer. Springer-Verlag Berlin Heiderberg 2001).

All animal experimentation procedures were performed in compliance with the guidelines of the institutional animal care committee of Shinshu University and the experiments were approved by the committee.

Statistical analysis. Survival rates were calculated using the Kaplan-Meier method and tested by the log-rank test. The tumor occurrence was tested by Fisher's direct method. All statistical analyses were performed using statistical software (Statcel, OMS, Tokorozawa, Japan), and p values of less than 0.05 were considered significant.

1. Oberlin, A., Endo, M. \& Koyama, T. Filamentous growth of carbon through benzene decomposition. J. Sryst. Growth 32, 335-349 (1976).

2. Iijima, S. Helical microtubules of graphitic carbon. Nature 354, 56-58 (1991).

3. Qu, L., Dai, L., Stone, M., Xia, Z. \& Wang, Z. L. Carbon nanotube arrays with strong shear binding-on and easy normal lifting-off. Science 322, 238-242 (2008).

4. Shannon, M. A. et al. Science and technology for water purification in the coming decades. Nature 452, 301-310 (2008).

5. Xiong, F., Liao, A. D., Estrada, D. \& Pop, E. Low-power switching of phase-change materials with carbon nanotube electrodes. Science 332, 68-570 (2011)

6. Van Noorden, R. Chemistry: the trials of new carbon. Nature 469, 14-16 (2011).
7. Endo, M. et al. Vapor-grown carbon fibers (VGCFs) basic properties and their battery applications. Carbon 39, 1287-1297 (2001).

8. Kang, S. J. et al. High-performance electronics using dense, perfectly aligned arrays of single-walled carbon nanotubes. Nat. Nanotechnol. 2, 230-236 (2007).

9. Scrosati, B. Nanomaterials: paper powers battery breakthrough. Nat. Nanotechnol. 2, 598-599 (2007).

10. Pugno, N. M., Bosia, F. \& Carpinteri, A. Multiscale stochastic simulations for tensile testing of nanotube-based macroscopic cables. Small 4, 1044-1052 (2008).

11. Byrne, M. T. \& Gun'ko, Y. K. Recent advances in research on carbon nanotubepolymer composites. Adv. Mater. 22, 1672-1688 (2010).

12. Pagona, G. \& Tagmatarchis, N. Carbon nanotubes: materials for medicinal chemistry and biotechnological applications. Curr. Med. Chem. 13, 1789-1798 (2006).

13. Saito, N. et al. Carbon nanotubes for biomaterials in contact with bone. Curr. Med. Chem. 15, 523-527 (2008).

14. Saito, N. et al. Carbon nanotubes: biomaterial applications. Chem. Soc. Rev. 38 , 1897-1903 (2009).

15. Harrison, B. S. \& Atala, A. Carbon nanotube applications for tissue engineering Biomaterials 28, 344-353 (2007).

16. Dubin, R. A., Callegari, G., Kohn, J. \& Neimark, A. Carbon nanotube fibers are compatible with Mammalian cells and neurons. IEEE Trans Nanobioscience 7. 11-14 (2008).

17. Usui, Y. et al. Carbon nanotubes with high bone-tissue compatibility and boneformation acceleration effects. Small 4, 240-246 (2008).

18. Lee, H. H., Sang Shin, U., Lee, J. H. \& Kim, H. W. Biomedical nanocomposites of poly(lactic acid) and calcium phosphate hybridized with modified carbon nanotubes for hard tissue implants. J. Biomed. Mater. Res. B Appl. Biomater. 98B, 246-254 (2011).

19. Shi Kam, N. W., Jessop, T. C., Wender, P. A. \& Dai, H. Nanotube molecular transporters: internalization of carbon nanotube-protein conjugates into mammalian cells. J. Am. Chem. Soc. 126, 6850-6851 (2004).

20. Yang, F. et al. Magnetic functionalised carbon nanotubes as drug vehicles for cancer lymph node metastasis treatment. Eur. J. Cancer. 47, 1873-1882 (2011).

21. Muller, J. et al. Respiratory toxicity of multi-wall carbon nanotubes. Toxicol. Appl. Pharmacol. 207, 221-231 (2005).

22. Lam, C. W., James, J. T., McCluskey, R., Arepalli, S. \& Hunter, R. L. A review of carbon nanotube toxicity and assessment of potential occupational and environmental health risks. Crit. Rev. Toxicol. 36, 189-217 (2006).

23. Carrero-Sanchez, J. C. Biocompatibility and toxicological studies of carbon nanotubes doped with nitrogen. Nano Lett. 6, 1609-1616 (2006).

24. Poland, C. A. Carbon nanotubes introduced into the abdominal cavity of mice show asbestos-like pathogenicity in a pilot study. Nat. Nanotechnol. 3, 423-428 (2008).

25. Ma-Hock, L. et al. Inhalation toxicity of multiwall carbon nanotubes in rats exposed for 3 months. Toxicol. Sci. 112, 468-481 (2009).

26. Auffan, M. et al. Towards a definition of inorganic nanoparticles from an environmental, health and safety perspective. Nat. Nanotechnol. 4. 634-641 (2009).

27. Oyabu, T. et al. Biopersistence of inhaled MWCNT in rat lungs in a 4-week well-characterized exposure. Inhal. Toxicol. 23, 784-791 (2011).

28. Nagai, H. et al. Diameter and rigidity of multiwalled carbon nanotubes are critical factors in mesothelial injury and carcinogenesis. Proc. Natl. Acad. Sci. USA 108, E1330-1338 (2011)

29. Porter, D. W. et al. Mouse pulmonary dose- and time course-responses induced by exposure to multi-walled carbon nanotubes. Toxicology 269, 136-147 (2010).

30. Kobayashi, N. et al. Biological response and morphological assessment of individually dispersed multi-wall carbon nanotubes in the lung after intratracheal instillation in rats. Toxicology 276, 143-153 (2010).

31. ISO 10993-1:2009 Biological evaluation of medical devices Part 1: Evaluation and testing.

32. van der Zande, M., Junker, R., Walboomers, X. F. \& Jansen, J. A. Carbon nanotubes in animal models: a systematic review on toxic potential. Tissue Eng. Part B Rev. 17, 57-69 (2011).

33. Long, G. G., Morton, D., Peters, T., Short, B. \& Skydsgaard, M. Alternative mouse models for carcinogenicity assessment: industry use and issues with pathology interpretation. Toxicol. Pathol. 38, 43-50 (2010).

34. Palazzi, X. \& Kergozien-Framery, S. Use of rasH2 transgenic mice for carcinogenesis testing of medical implants. Exp. Toxicol. Pathol. 61, 433-441 (2009).

35. Hara, K. et al. Evaluation of CNT toxicity in comparison to tattoo ink nanoparticles for use as a biomaterial. Mater. Today 14, 434-440 (2011)

36. Urano, K. et al. Examination of percutaneous application in a 26 -week carcinogenicity test in CB6F1-TG rasH2 mice. J. Toxicol. Sci. 32, 367-375 (2007).

37. Firme, 3rd, C. P. \& Bandaru, P. R. Toxicity issues in the application of carbon nanotubes to biological systems. Nanomedicine 6, 245-256 (2010).

38. Beg, S. et al. Advancement in carbon nanotubes: basics, biomedical applications and toxicity. J. Pharm. Pharmacol. 63, 141-163 (2011).

39. Shvedova, A. A. et al. Mechanisms of pulmonary toxicity and medical applications of carbon nanotubes: Two faces of Janus? Pharmacol. Ther. 121, 192-204 (2009).

40. Yang, K. \& Liu, Z. In vivo biodistribution, pharmacokinetics, and toxicology of carbon nanotubes. Curr. Drug Metab. (2012) [Epub ahead of print] 
41. Wang, L. et al. Dispersion of single-walled carbon nanotubes by a natural lung surfactant for pulmonary in vitro and in vivo toxicity studies. Part. Fibre Toxicol. 7, $31(2010)$

42. Palomäki, J. et al. Long, needle-like carbon nanotubes and asbestos activate the NLRP3 inflammasome through a similar mechanism. ACS Nano 27, 6861-6870 (2011).

43. Patlolla, A. K., Berry, A. \& Tchounwou, P. B. Study of hepatotoxicity and oxidative stress in male Swiss-Webster mice exposed to functionalized multi-walled carbon nanotubes. Mol. Cell Biochem. 358, 189-199 (2011).

44. Sanchez, V. C., Weston, P., Yan, A., Hurt, R. H. \& Kane, A. B. A 3-dimensional in vitro model of epithelioid granulomas induced by high aspect ratio nanomaterials. Part. Fibre Toxicol. 8, 17 (2011).

45. Teeguarden, J. G. et al. Comparative proteomics and pulmonary toxicity of instilled single-walled carbon nanotubes, crocidolite asbestos, and ultrafine carbon black in mice. Toxicol. Sci. 120, 123-135 (2011).

46. Ando, K. et al. Chemically induced forestomach papillomas in transgenic mice carry mutant human c-Ha-ras transgenes. Cancer Res. 52, 978-982 (1992).

47. Boverhof, D. R. et al. Transgenic animal models in toxicology: historical perspectives and future outlook. Toxicol Sci. 121, 207-233 (2011).

48. Urano, K., Tamaoki, N. \& Nomura, T. Establishing a laboratory animal model from a transgenic animal: $\mathrm{ras} \mathrm{H} 2$ mice as a model for carcinogenicity studies in regulatory science. Vet. Pathol. 49, 16-23 (2012).

49. Urano, K. et al. Use of IC tags in short-term carcinogenicity study on CB6F1 TGrasH2 mice. J. Toxicol. Sci. 31, 407-418 (2006).

50. Palazzi, X. \& Kergozien-Framery, S. Use of rasH2 transgenic mice for carcinogenesis testing of medical implants. Exp. Toxicol. Pathol. 61, 433-441 (2009).

51. Di Sotto, A., Chiaretti, M., Carru, G. A., Bellucci, S. \& Mazzanti, G. Multi-walled carbon nanotubes: Lack of mutagenic activity in the bacterial reverse mutation assay. Toxicol. Lett. 184, 192-197 (2009).

52. Thurnherr, T. et al. A comparison of acute and long-term effects of industrial multiwalled carbon nanotubes on human lung and immune cells in vitro. Toxicol. Lett. 200, 176-186 (2011).

53. Naya, M. et al. Evaluation of the genotoxic potential of single-wall carbon nanotubes by using a battery of in vitro and in vivo genotoxicity assays. Regul. Toxicol. Pharmaco. 61, 192-198 (2011).
54. Sargent, L. M., Reynolds, S. H. \& Castranova, V. Potential pulmonary effects of engineered carbon nanotubes: in vitro genotoxic effects. Nanotoxicology 4 , 396-408 (2010).

\section{Acknowledgements}

We thank M. Iwaya, Y. Ozawa, and M. Maruyama for help at the different stages of the project. This research was supported by the Regional Innovation Cluster Program (the second stage) of the Ministry of Education, Culture, Sports, Science and Technology, Japan, by a Grant-in-Aid for Specially Promoted Research from the Ministry of Education, Science, Sports, and Culture, Japan (No. 19002007), by the Research and Development of Nanodevices for Practical Utilization of Nanotechnology of the New Energy and Industrial Technology Development Organization, Japan, and by Japan Regional Innovation Strategy program by the Excellence of the Japan Science and Technology Agency, and Hospital-company collaboration support project for developing/improving problem-solving-type medical equipment by Ministry of Economy, Trade and Industry, Japan.

\section{Author contributions}

S.T., K.H., K.A., H.T., K.M. and N.S. designed the study, analysed the data and wrote the manuscript. S.T., K.H., K.A., Y.U., M.S., H.H., N.O., K.N., S.K., K.S. and N.N. performed experiments. N.I. and M.O. contributed to discussions. H.K. supervised the project. All Authors discussed the results and commented on the manuscript.

\section{Additional information}

Supplementary information accompanies this paper at http://www.nature.com/ scientificreports

Competing financial interests: The authors declare no competing financial interests.

License: This work is licensed under a Creative Commons

Attribution-NonCommercial-ShareAlike 3.0 Unported License. To view a copy of this license, visit http://creativecommons.org/licenses/by-nc-sa/3.0/

How to cite this article: Takanashi, S. et al. Carcinogenicity evaluation for the application of carbon nanotubes as biomaterials in rasH2 mice. Sci. Rep. 2, 498; DOI:10.1038/srep00498 (2012). 


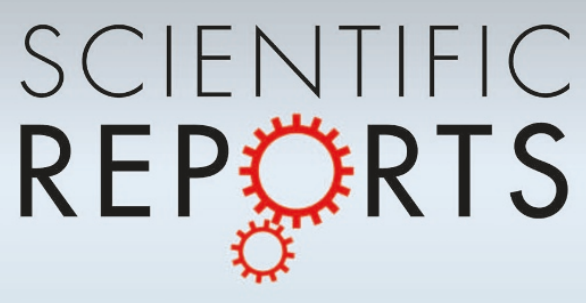

DOI: 10.1038/srep01688

SUBJECT AREAS: BIOMATERIALS CARBON NANOTUBES AND FULLERENES TOXICOLOGY ONCOGENESIS

SCIENTIFIC REPORTS: $2: 498$ DOI: $10.1038 /$ srep00498 (2012)

Published: 9 July 2012

Updated: 17 April 2013
CORRIGENDUM: Carcinogenicity evaluation for the application of carbon nanotubes as biomaterials in ras $\mathrm{H} 2$ mice

Seiji Takanashi, Kazuo Hara, Kaoru Aoki, Yuki Usui, Masayuki Shimizu, Hisao Haniu, Nobuhide Ogihara, Norio Ishigaki, Koichi Nakamura, Masanori Okamoto, Shinsuke Kobayashi, Hiroyuki Kato, Kenji Sano, Naoyuki Nishimura, Hideki Tsutsumi, Kazuhiko Machida \& Naoto Saito

The authors have noticed that Figure $4 \mathrm{C}$ was published incorrectly in the original version of this Article. The correct Figure 4C appears below in Figure 1.

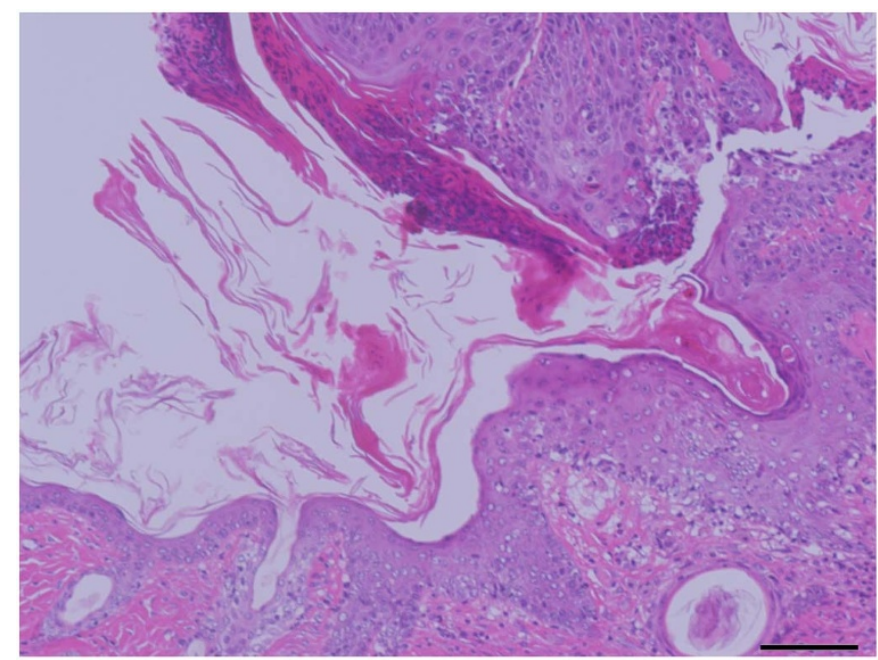

